vorauszudenken. Als Mittel für erfolgreiches Kostenmanagement werden bei der Henkel AG \& Co. KGaA Shared Service Center eingesetzt. Dr. Marcus Kuhnert, Financial Director der Laundry \& Home Care Division, beschrieb die Entwicklung und zeigte Beispiele, bevor Peter Kuhl, Geschäftsführer (CFO) der uhlsport $\mathrm{GmbH}$, mit seinem Unternehmen ein weiteres Fallbeispiel anführte. Von eigenen Erfahrungen geprägt, warb er vor allem dafür, den strategischen Fokus bei Kostensenkungsmaßnahmen nicht außer Acht zu lassen. Vorträge und Diskussionen zu den Themenbereichen Compliance, Risiko- management sowie dem Verhältnis von CFO und Investoren schlossen den Kreis der Beiträge.

Der 9. Handelsblatt CFO-Kongress fand am 20. und 21. Juni 2013 in Königstein im Taunus statt. Das umfassende Vortragsprogramm wurde abgerundet durch zahlreiche Möglichkeiten zum Netzwerken und Austauschen.

Weitere Informationen zu der Veranstaltung finden Sie im Internet unter www.cfo-kongress.de.

Simone Schwenninger, Vallendar

\title{
Forschungsnachwuchs trifft Top-Forscher zwischen Prater und Heurigem
}

\section{Bericht von der Empirical Research in Management, Accounting \& Control Konferenz (ERMAC) in Wien}

Das Institut für Unternehmensführung, welches die ERMACKonferenz organisiert, legt traditionell großen Wert auf den Dialog zwischen Wissenschaft und Praxis. So liegt der generelle Fokus der Konferenz explizit auf der Diskussion von Anwendungen und Methoden der Controlling-Forschung im Bereich der Empirie. Das illustre Feld von aufstrebenden Nachwuchsforschern wurde in diesem Jahr durch Top-Forscher wie Sim B. Sitkin, Frank Moers und Teemu Malmi ergänzt. Hierzu gesellte sich mit Ken Merchant eine wahre Koryphäe auf dem Gebiet der empirischen Feldforschung. Dieser präsentierte im Rahmen seiner Keynote Speech seine aktuellen Gedanken hinsichtlich Feldforschung und Ethik. Seiner Ansicht nach ist eines der Hauptprobleme der empirischen Feldforschung, dass sich ihre Anwendung häufig als sehr kompliziert und zeitaufwendig darstellt. Um diesem Manko entgegenzuwirken, schlägt er vor, statt der bisher verwendeten - äußerst umfangreichen - reinen Feldstudien vermehrt Archivdatenstudien durchzuführen und diese durch kurze Feldstudienkomponenten zu ergänzen. Darüber hinaus rief er dazu auf, in zukünftigen Studien den Einfluss von Ethik auf Management-Control-Systeme (MCS) stärker zu berücksichtigen. Seiner Meinung nach sind Ethik- bzw. ComplianceProgramme und MCS, obwohl sie häufig in Unternehmen als verschiedene Dinge angesehen werden, eigentlich nichts anderes als Komplemente zueinander.

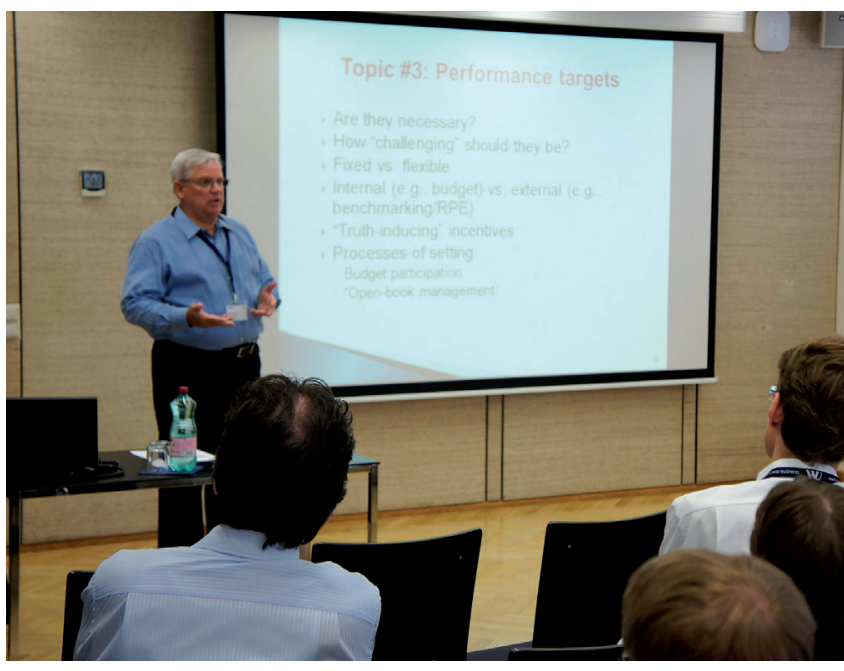

Ken Merchant präsentiert seine Ansätze und Ideen.

Zum dritten Mal fand die ERMAC-Konferenz dieses Jahr im großen Sitzungssaal der Wirtschaftsuniversität Wien statt. Neben spannenden Vorträgen und Diskussionen wurde die Konferenz von einem ausgezeichneten Rahmenprogramm abgerundet, welches den obligatorischen Besuch eines Wiener Heurigen natürlich nicht vermissen ließ.

Weitere Informationen zu dieser Veranstaltung finden Sie im Internet unter www.wu.ac.at/ifu/conference.

Florian Herschung, Vallendar 